\title{
25 Jahre Forum der Psychoanalyse
}

\section{Michael Ermann}

Online publiziert: 3. November 2009

(C) Springer-Verlag 2009

Vor über 25 Jahren, wir schrieben noch das Jahr 1984, machten Jürgen Körner und ich einen langen Spaziergang durch die Weinberge an der badischen Bergstraße, vertieft in Gedanken über die Lage und Zukunft der Psychoanalyse in Deutschland.

Damals trugen die deutschen Psychoanalytiker noch schwer am unglückseligen Erbe aus der Zeit des Nationalsozialismus. Der Ausschluss der jüdischen Psychoanalytiker aus der psychoanalytischen Fachgesellschaft, das Schweigen zu deren Verfolgung und Emigration und die Mitarbeit im nationalsozialistisch dominierten psychotherapeutischen „Reichsinstitut", in dem die Freudsche Lehre verfemt und die Neopsychoanalyse zumindest geduldet wurde, all das lastete auf der psychoanalytischen Gemeinschaft. Sie war in Fraktionen gespalten, die einander wenig beachteten. Eine Gruppe, die Deutsche Psychoanalytische Vereinigung (DPV), war eng mit der Internationalen Psychoanalytischen Vereinigung (IPV) verbunden, die andere, die Deutsche Psychoanalytische Gesellschaft (DPG), hatte sich lange an der Neopsychoanalyse orientiert und war von den internationalen Entwicklungen weitgehend abgeschnitten geblieben. Dazwischen gab es regionale Gruppierungen, die versuchten, eigene Wege zu gehen.

Als Funktionsträger in der DPG ${ }^{1}$ waren wir angesichts zunehmender Kritik an der Psychoanalyse besorgt über die Schwächung der Kräfte durch die Spannungen, die das Klima damals beherrschten. So suchten wir nach Wegen, ein konstruktives Klima zu schaffen und die Kluft zwischen den Gruppen zu mäßigen. Dabei ließen wir uns auch davon leiten, dass mit dem Beginn der 1980er Jahre eine Besinnung auf die gemeinsame Geschichte der Psychoanalytiker in Deutschland begonnen hatte. Es schien sich eine Anerkennung der gemeinsamen Verantwortung für die Entwicklungen in der NS-Zeit anzukündigen, auf die sich unsere Hoffnung richtete.

Vor diesem Hintergrund gelangten Körner und ich zu dem Plan, die Aufweichung der Fraktionsbildungen zu nutzen und durch die Gründung einer wissenschaftlichen Zeitschrift

Prof. Dr. med. M. Ermann $(\bowtie)$

München, Deutschland

E-Mail: Ermann-Forum@t-online.de

\footnotetext{
${ }^{1}$ Damals war ich Vorsitzender der DPG, Jürgen Körner Stellvertreter.
} 
zu fördern, die allen Fachgesellschaften offenstehen sollte und nicht einem der psychoanalytischen Vereine verpflichtet war.

Das Ergebnis dieses Spaziergangs war der spontane Entschluss, Sven Olaf Hoffmann, prominentes Mitglied der DPV, in Mainz aufzusuchen. Über die Zeitschrift Praxis der Psychotherapie und Psychosomatik bestand zu ihm eine freundschaftlich-kollegiale Verbindung. Er gehörte zu jenen, die die Vereinspolitik der Fachgesellschaften mit souveräner Distanz verfolgten und zugleich für die Belange der Psychoanalyse in Theorie und Praxis eintraten. Er ließ sich sofort für das Projekt gewinnen, und so entwarfen wir noch am selben Abend das „Programm“:

Das Forum der Psychoanalyse ist eine Zeitschrift für die Psychoanalyse als klinische Theorie und Praxis und als Grundlagenwissenschaft. Es steht allen Psychoanalytikern offen. Das Forum dient der Diskussion der verschiedenen Strömungen in der Psychoanalyse, auch in Auseinandersetzung mit benachbarten Wissenschaften. Es fördert den Austausch zwischen den Fachgesellschaften und ist eine Brücke zu den Entwicklungen der Psychoanalyse im Ausland.

Auch der Verantwortliche im Springer-Verlag für die Zeitschrift Praxis, Toni Graf-Baumann, fand rasch Gefallen an dem Projekt und zerstreute unsere (damals sicher berechtigten) Bedenken und Zweifel, ob eine Neugründung sich neben der mächtigen Psyche behaupten würde. Mühelos ließ sich ein ansehnliches Herausgeber- und Beiratsgremium gewinnen, das unsere Idee einer Brücke zwischen den Fachgesellschaften und ins Ausland hervorragend repräsentierte (Abb. 1). Wir waren selbst überrascht, offenbar die Zeichen der Zeit und den Trend in Richtung einer vorsichtigen Annäherung richtig gedeutet zu haben.

Die Resonanz, die das Projekt gleich mit dem Erscheinen des ersten Heftes im Juli 1985 hervorrief, war ermutigend. Die Gründungsidee fand dauerhaften Widerhall in einer rasch wachsenden Leserschaft. Der Schwerpunkt bei klinischen Themen aus der Sicht der verschiedenen zeitgenössischen Strömungen und der Traditionen der verschiedenen Fachgesellschaften erweckte ihr Interesse. Dankbar wurde die Bereitschaft renommierter Autoren aus dem In- und Ausland aufgenommen, im Forum zu publizieren. Auch die Einführung von Rubriken wie dem „Aktuellen Forum“ und dem „Forum moderner Klassiker“ fand das Interesse des Publikums. So war schon mit dem dritten Jahrgang ein Abonnentenstamm gewonnen, der das Projekt auch wirtschaftlich trug. Dieser Erfolg konnte bis heute ohne bedeutende Krisen fortgesetzt werden.

Mit dem zehnten Jahrgang verabschiedete sich Sven Olaf Hoffmann aus der Schriftleitung. Carl Nedelmann trat an seine Stelle. Mit dem 24. Jahrgang kamen Anna Ursula Dreher und Ulrich Lamparter hinzu. Gleichzeitig wurde das Board umstrukturiert, sodass die bisherige Schriftleitung zum Herausgebergremium wurde und die bisherigen Herausgeber, von denen die meisten die Zeitschrift von Beginn an mitgetragen hatten, in den Beirat eintraten.

Ein Garant für die Kontinuität im Erscheinen der Hefte und die verlegerische Qualität der Beiträge war die unermüdliche und fürsorgliche Arbeit unserer Redakteurin, Regine Karcher-Reiners, die die Zeitschrift seit über zwei Jahrzehnten betreut. Veränderungen gab es dagegen aufseiten des Verlages: Toni Graf-Baumann verließ den Springer-Verlag und übergab das Forum an Heike Berger. Mit dem 22. Band kam die Zeitschrift an den SpringerTochterverlag Steinkopff und dort in die Obhut von Sabine Ibkendanz. Sie alle sorgten dafür, dass der Stil trotz Veränderungen im äußeren Erscheinungsbild, die durch die moderne Verlagstechnik und die Onlinepublikation erforderlich wurden, bewahrt werden konnte.

Mit dem vorliegenden Heft beschließen wir den 25. Jahrgang. Der Verlag hat zu diesem Anlass eine CD-ROM beigesteuert, die ein Gesamtinhalts- und komplettes Sachverzeichnis 


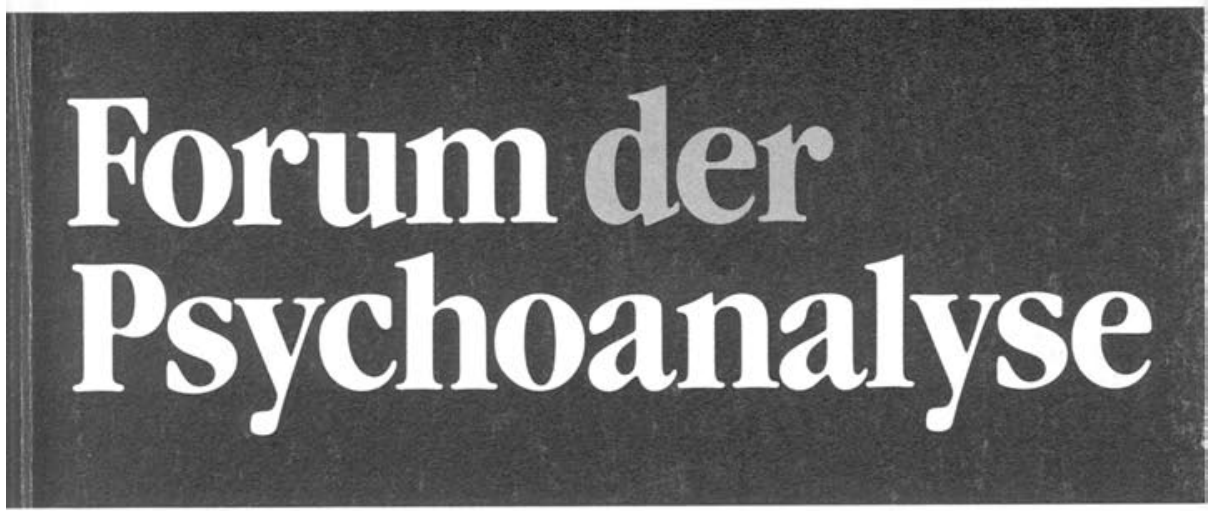

Band 1 Heft 1 Juli 1985

M. Ermann Editorial. Forum der Psychoanalyse 1

\section{Originalarbeiten}

H. Thomä, H.Schrenk, Der psychoanalytische Dialog und die Gegenfrage-Regel

H. Kächele The psychoanalytic dialogue and the counterquestion rule 4

M. H. Stein Kommentar zur vorstehenden Arbeit 18

J.Körner, U.Rosin Das Problem der Abstinenz in der Psychoanalyse

The problem of abstinence in psychoanalysis 25

B. Grunberger Narziß und Anubis

Narciss and Anubis 48

S. O. Hoffmann Psychoanalyse im ,Spiegel“" 60

\section{Diskussionsforum}

\section{Aktuelles Forum}

J.Kafka Gedanken zur Begegnung in Deutschland 72

Tagungsbericht

G. Schmidt Arbeitstagung der Deutschen Psychoanalytischen Vereinigung 75

Mitteilungen - Autoren dieses Heftes 79

\section{Springer-Verlag}

Berlin Heidelberg New York Tokyo

451 Forum Psychoanal (1985) 1 (1) 1-80

Abb. 1 Titelblatt der ersten Ausgabe der Zeitschrift im Jahre 1985 sowie Herausgebergremium des ersten Bandes 

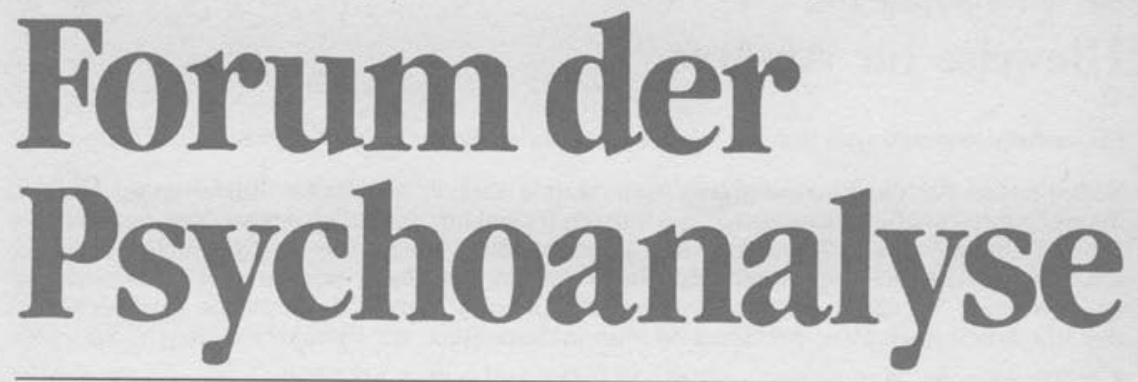

\section{Herausgeber}

Friedrich Beese, Horb Tobias Brocher, Neuss Helmut Enke, Stuttgart Michael Ermann, München Peter Fürstenau, Düsseldorf Annelise Heigl-Evers, Düsseldorf
Sven Olaf Hoffmann, Mainz Horst Kächele, UIm Karl König, Göttingen Jürgen Körner, Bad Berleburg Emma Moersch, Frankfurt Theodor Seifert, Stuttgart

\section{Schriftleitung}

Michael Ermann, Sven Olaf Hoffmann und Jürgen Körner

\section{Wissenschaftlicher Beirat}

Gaetano Benedetti, Basel - Horus-Vital Brazil, Rio de Janeiro - Arthur H. Feiner, New York · Béla Grunberger, Paris · John Kafka, Washington Jean G. Lemaire, Paris - Lester Luborsky, Philadelphia - Malcolm Pines, London · Ludwig Reiter, Wien · Hans Strotzka, Wien sowie Hannes Friedrich, Göttingen · Paul L. Janssen, Essen Christa Rohde-Dachser, Hannover · Ulrich Streeck, Göttingen

\section{Springer-Verlag Berlin Heidelberg New York Tokyo}

(Fortsetzung) 
und je einen repräsentativen Beitrag pro Band enthält. Sie soll dem Leser die Orientierung erleichtern und kündigt vielleicht schon das Medium der Zukunft an.

Sicherlich hat das Profil des Forums sich über die Jahre mit den jeweils aktuellen Themen verändert. Neue Rubriken wie das Ausbildungs- und das Traumforum, das Vortrags- und das Ost-West-Forum sind hinzugekommen. Wir waren bemüht, dadurch Schwerpunktsetzungen noch deutlicher zu machen und die Leser noch gezielter anzusprechen. Im Kern bleibt aber die Idee leitend, die klinische Praxis vor dem Hintergrund einer Theorienlandschaft zu reflektieren, die sich in einem raschen Wandel befindet. Dabei möglichst verschiedene Ansichten und Auffassungen zu Wort kommen zu lassen, wird auch in Zukunft das Gesicht des Forums prägen.

Nach 25 Jahren hat sich aber auch die Landschaft der institutionalisierten Psychoanalyse in Deutschland verändert. Die Entwicklung dieser zweieinhalb Jahrzehnte lässt sich an zwei Beiträgen ablesen: Im Jahr 1985 beendeten wir den ersten Jahrgang mit Klaus von Dohnanyis Rede vor dem ersten IPV-Kongress nach dem Krieg in Deutschland, der von der DPV in Hamburg ausgerichtet worden war. Er stand noch ganz unter dem Eindruck von Schuld und Scham im Zusammenhang mit der nationalsozialistischen Vergangenheit. In diesem Heft, 25 Jahre später, finden Sie die Dankesrede, mit der sich Franz Wellendorf beim IPV-Kongress 2009 in Chicago für die Wiederaufnahme der DPG in die IPV bedankt. Dazwischen liegen Jahre eines mühsamen, anstrengenden und engagierten Prozesses innerhalb und zwischen den psychoanalytischen Gruppierungen mit dem Ergebnis, dass die Wunden der NS-Zeit vernarben und die ehemals zerklüftete Landschaft der Psychoanalyse in Deutschland sich normalisiert.

Als Herausgeber, Beirat, Redaktion und Verlag des Forum der Psychoanalyse sind wir stolz und dankbar, zu diesem Prozess einen Beitrag geleistet zu haben.

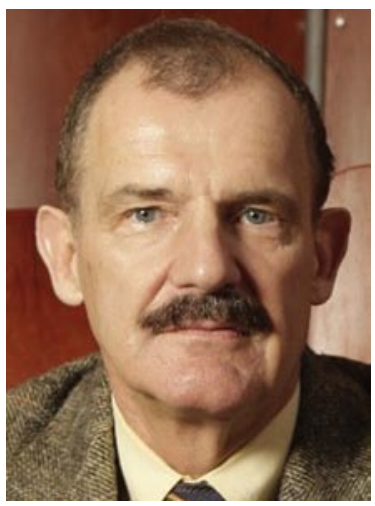

Michael Ermann, Prof. Dr. med., Jg. 1943, Facharzt für psychosomatische Medizin und Psychotherapie sowie Psychoanalytiker (IPV/DPG). Er leitete von 1985 bis 2009 die Abteilung für Psychosomatik und Psychotherapie der Psychiatrischen Klinik der Ludwig-Maximilians-Universität. 1985 gründete er zusammen mit Sven O. Hoffmann und Jürgen Körner diese Zeitschrift. 